\subsection{7.-24.07.2004 \\ Zürich}

Schweiz

\subsection{9.-04.09.2004}

Wilsede

Deutschland

\section{[................. \\ 02.09.-04.09.2004}

Freiburg i.Br.

Deutschland

\subsection{9.-21.09.2004 \\ Köln}

Deutschland

\section{International Skin Cancer Conference}

Wilsede-Schule für Onkologie und Hämatologie: Mikroskopierkurs für Anfänger

\title{
24. Jahrestagung der Deutschen Gesellschaft für Senologie
}

6. International Symposium on Hodgkin's Lymphoma

\subsection{9.-25.09.2004 \\ Basel}

Schweiz
Senologie Kongress 2004

Gemeinsame Jahrestagung der Österreichischen und Schweizerischen

Gesellschaften für Senologie

"Erkrankungen der Brustdrüse - eine interdisziplinäre Herausforderung»

\subsection{9.-03.10.2004}

UIm

Deutschland

\section{World Congress Self-Care Deficit Nursing Theory} S-DCNT

Die Wissenschaft der Selbstpflege und ihre Bedeutung für Pflegeforschung, Praxis, Management und Ausbildung

Gastrointestinale Tumoren + Update Mammakarzinom 2004
Auskunft: Reinhard Dummer, M.D.

Dept. of Dermatology

University Hospital Zürich

Gloriastr. 31, CH-8091 Zürich

Tel. +41 1 255-8837, Fax -4403

E-mail nicole.brunner@usz.ch

www.skincancer.ch

Auskunft: Frau Klara Schlunk

Klinikum Oldenburg

Klinik für Innere Medizin II

Dr. Eden Str. 10

D-26133 Oldenburg

Tel. +49 441 403-2611, Fax -2654

E-mail illiger.h.j@klinikum-oldenburg.de

Auskunft: CTW - Congress Organisation

Thomas Wiese GmbH

Goßlerstr. 30

D-12161 Berlin

Tel. +49 30 85-996216, Fax -079826

E-mail senologie@ctw-congress.de

Auskunft: Clinic I for Internal Medicine

University Clinic of Cologne

Congress secretariat (6th ISHL)

Haus LebensWert

Joseph-Stelzmann-Str. 9

D-50924 Köln

Tel. +49 221 478-86148, Fax -86654

E-mail hodgkin2004@medizin.uni-koeln.de

www.hodgkin2004.de

Auskunft: Administratives Sekretariat

Senologie 2004

c/o AKM Congress Service

Clarastr. 57 / Postfach

CH-4005 Basel

Tel. +41 61 68677-11, Fax -88

E-mail info@akm.ch

Auskunft: EMTS - European Medical Trial

Support GmbH

Brühlstr. 19

D-89081 Ulm

Tel. +497316023576

www.worldcongress-scdnt.com

Auskunft: European School of Oncology

Deutschsprachiges Programm (ESO-D)

Frau Doris Hoevel

Rorschacherstr. 150

CH-9006 St. Gallen

Tel. +41 71-243 0032, Fax -245 6805

E-mail eso-d@sg.zetup.ch

Auskunft: Mondial Congress

Faulmanngasse 4

A-1040 Wien

Tel. +43 1-588 040, Fax -586 9185

E-mail dgho-ögho2004@mondial.at

\section{KARGER}




\subsection{0.-09.10.2004}

Wiesbaden

Deutschland

\subsection{0.-14.10.2004}

Wien

Österreich

\section{- $\ldots \ldots \ldots \ldots \cdots \cdots$}

15.10.-16.10.2004

Wien

Österreich

[................

21.10.-23.10.2004

Freiburg i.Br.

Deutschland

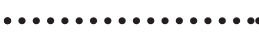

21.10.-23.10.2004

München

Deutschland

\subsection{0 .02 .112004}

Wien

Österreich

•....................

26.01.-29.01.2005

St. Gallen

Schweiz
18.02.-19.02.2005

Aachen

Deutschland

\section{ÖGRO 2004}

21. Jahrestagung der Österreichischen Gesellschaft für Radioonkologie, Radiobiologie und medizinische Radiophysik

5. International Freiburg Symposium on Molecular Hematology

Jahrestagung der Arbeitsgemeinschaft Dermatologische Onkologie (ADO)

\section{ESMO Congress}

9. International Conference on Primary Therapy of Early Breast Cance

\section{AGO-Winterkurs}

Metastasierung gastrointestinaler Tumoren
Auskunft: EMTS - European Medical Trial

Support GmbH

Brühlstr. 19

D-89081 Ulm

Tel. +497316023576

www.bic-conference.de

Auskunft: ÄRZTEZENTRALE MED.INFO

Helferstorferstr. 4

A-1014 Wien

Tel. +431 531 16-38, Fax -61

E-mail azmedinfo@media.co.at

www.oegro2004wien.at

Auskunft: ÄRZTEZENTRALE MED.INFO

Helferstorferstr. 4

A-1014 Wien

Tel. +43 1531 16-38, Fax -61

E-mail azmedinfo@media.co.at

www.oegro2004wien.at

Auskunft: Prof. H. Veelken

Medizinische Klinik I

Universitätsklinikum Freiburg

Hugstetter Str. 55

D-79106 Freiburg

Tel. +49 761 270-3462 / 3530, Fax -3233

E-mail veelken@mm11.ukl.uni-freiburg.de

•..............................

Auskunft: Prof. Dr. Matthias Volkenandt

Dermatologische Klinik der LMU

Frauenlobstr. 9

D-80337 München

Tel. +498951606225

www.ado-homepage.de

Auskunft: ESMO

Congress Secretariat

Via La Santa 7

CH-6962 Viganello-Lugano

Tel. +41 91 950-0781, Fax -0782

E-mail alessia@esmo.org,chatrina@esmo.org

www.esmo.org

Auskunft: St. Gallen Oncology Conferences

Frau Beatrice Nair

Rorschacherstr. 150, POB

CH-9006 St. Gallen

Tel. +41 71-243 0032, Fax -245 6805

E-mailinfo@oncoconferences.ch

www.oncoconferences.ch

Auskunft: Herrn Prof. Dr. med. S. Mater

Medizinische Klinik III des Universitätsklinikums der RWTH Aachen

Pauwelsstr. 30

D-52074 Aachen

Tel. +49 241 80-88634, Fax -82455

Emailmk3@ukaachen.de 